\title{
Autologous implantation of bilayered tissue-engineered respiratory epithelium for tracheal mucosal regenesis in a sheep model.
}

\begin{abstract}
The objective of this study was to regenerate the tracheal epithelium using autologous nasal respiratory epithelial cells in a sheep model. Respiratory epithelium and fibroblast cells were harvested from nasal turbinates and cultured for 1 week. After confluence, respiratory epithelium and fibroblast cells were suspended in autologous fibrin polymerized separately to form a tissue-engineered respiratory epithelial construct (TEREC). A $3 \times 2 \mathrm{~cm} 2$ tracheal mucosal defect was created, and implanted with TEREC and titanium mesh as a temporary scaffold. The control groups were divided into 2 groups: polymerized autologous fibrin devoid of cells (group 1), and no construct implanted (group 2). All sheep were euthanized at 4 weeks of implantation. Gross observation of the trachea showed minimal luminal stenosis formation in the experimental group compared to the control groups. Macroscopic evaluation revealed significant mucosal fibrosis in control group $1(71.8 \%)$ as compared to the experimental group (7\%). Hematoxylin and eosin staining revealed the presence of minimal overgrowth of fibrous connective tissue covered by respiratory epithelium. A positive red fluorescence staining of PKH26 on engineered tissue 4 weeks after implantation confirmed the presence of cultured nasal respiratory epithelial cells intercalated with native tracheal epithelial cells. Scanning electron microscopy showed the presence of short microvilli representing immature cilia on the surface of the epithelium. Our study showed that TEREC was a good replacement for a tracheal mucosal defect and was able to promote natural regenesis of the tracheal epithelium with minimal fibrosis. This study highlighted a new technique in the treatment of tracheal stenosis.
\end{abstract}

Keyword: Fibrin, autologous; Respiratory epithelium; Tissue engineering. 